1 Fundação Oswaldo Cruz

(Fiocruz), Escola Nacional de Saúde Pública Sergio Arouca (Ensp), Programa de Pós-Graduação em Saúde Pública (PPGSP) Rio de Janeiro (RJ), Brasil. marlucerodriguesenf@gmail. com

2 Fundação Oswaldo Cruz (Fiocruz), Escola Nacional de Saúde Pública Sergio Arouca (Ensp), Departamento de Direitos Humanos, Saúde e Diversidade Cultural (DIHS) - Rio de Janeiro (RJ), Brasil.

aldopachecoferreira@gmail. com

\section{Aposentadoria no contexto de Reforma Previdenciária: análise descritiva em uma instituição de ensino superior}

\author{
Retirement in the context of the Social Security Reform: descriptive
} analysis in a higher education institution

Marluce Rodrigues Godinho', Aldo Pacheco Ferreira²

RESUMO Visando a analisar o perfil sociodemográfico, de trabalho e saúde dos aposentados de uma universidade pública, foi realizado um estudo de coorte através de análise retrospectiva de dados dos aposentados. A maioria aposentou-se por idade ou tempo de serviço e apresentava boas condições de trabalho, saúde e boa capacidade para o trabalho. As condições favoráveis para a manutenção da vida ativa no trabalho desses indivíduos podem servir como exemplo para outras categorias profissionais no País. Sugere-se, então, pensar na Reforma Previdenciária junto a ações de prevenção de doenças e promoção da saúde, para prevenir o adoecimento, a perda da capacidade para o trabalho e as aposentadorias precoces.

PALAVRAS-CHAVE Saúde do trabalhador. Aposentadoria. Setor público. Previdência social.

ABSTRACT In order to analyze the sociodemographic, work and health profile of the retirees of a public university, a cohort study was carried out through a retrospective data analysis of the retirees. The majority retired by age or time of service and presented good working conditions, health and good work ability. The favorable conditions for the maintenance of the active life in the work of these individuals can serve as an example for other professional categories in the Country. It is suggested, then, to think about the Social Security Reform along with actions of prevention of diseases and health promotion to prevent the illness, the loss of work ability and early retirements.

KEYWORDS Occupational health. Retirement. Public sector. Social security. 


\section{Introdução}

Nas últimas décadas, o mundo vem passando por um processo de envelhecimento populacional, resultado do maior crescimento da população idosa quando comparado ao dos demais grupos etários (FREIRE; MENDONÇA; COSTA, 2012). Nesse contexto, o Japão é exemplar das sociedades em processo de envelhecimento no que diz respeito à longevidade e à proporção de idosos na população (AKIYAMA, 2015). Estima-se que, em 2030, um terço da população japonesa terá idade superior a 65 anos, sendo que $20 \%$ terão 75 anos de idade ou mais (AKIYAMA, 2015). Já no Brasil, o número de idosos ( $\geq 60$ anos de idade) passou de 3 milhões, em 1960, para 7 milhões, em 1975, e 14 milhões em 2002 (um aumento de 500\% em quarenta anos), e estima-se que alcançará 32 milhões em 2020 (LIMA-COSTA; VERAS, 2003).

Embora essa seja uma situação global, os impactos social e econômico desse processo se dão de maneiras diferentes nos países desenvolvidos e naqueles em desenvolvimento, como o Brasil. O fato é que, nos países menos desenvolvidos, o contingente de pessoas prestes a envelhecer, dadas as reduções das taxas de mortalidade, é proporcionalmente maior quando comparado com o contingente disponível no início do século. Com a baixa real da fecundidade, a tendência é de haver transformações significativas na estrutura etária desses países, em tempo relativamente curto, sem que as conquistas sociais tenham se processado devidamente para a maioria da população, ou seja, sem que tenha havido uma real melhoria das condições de vida de uma grande parcela dessas populações (BARILE ET AL., 2012; YORKSTON; BOURGEOIS; BAYLOR, 2010).

Considerando esse quadro, principalmente nos países em desenvolvimento, os governos estão estudando estratégias para evitar as aposentadorias precoces e prolongar a vida ativa, aumentando a idade para aposentadoria (ROBROEK ET AL., 2013). O Governo brasileiro, preocupado com as mudanças no perfil demográfico e diante da crise financeira que o País tem vivenciado, tem provocado discussões sobre o que vem sendo denominado de 'Reforma da Previdência'. Essa Reforma, oficializada através da Proposta de Emenda à Constituição (PEC 287), está em tramitação no Congresso e, se for aprovada, tal como apresentada, trará importantes modificações na previdência social, tais como: aumento da idade de aposentadoria voluntária para 65 anos, tanto para homens quanto para mulheres; o tempo mínimo de contribuição passará de 15 para 25 anos, sem que seja garantido o benefício completo, pois o trabalhador receberá apenas $76 \%$ do benefício, e, caso queira receber o benefício integral, deverá contribuir por 49 anos; aqueles que possuíam tempo de contribuição diferenciado, como agricultores familiares e professores, passarão a seguir a mesma regra de idade dos demais segurados; a pensão por morte passará de integral para $60 \%$ do benefício e mais $10 \%$ para cada dependente; não será mais permitido receber aposentadoria e pensão simultaneamente; e, ainda, como se não bastasse, a idade mínima de 65 anos poderá ser ampliada sem aviso prévio, de acordo com o aumento da expectativa de vida da população brasileira.

No entanto, é difícil se pensar em Reforma Previdenciária e saúde do trabalhador como temas díspares, pois, para que seja prolongado o tempo de contribuição e elevada a idade para aposentadoria, primeiramente, é necessário investir em melhores condições de trabalho e saúde para os trabalhadores, a fim de que eles possam permanecer no mercado de trabalho saudáveis e com satisfação. Akiyama (2015) relata que, se forem promovidas as condições certas, os idosos podem continuar a fazer parte da população ativa mesmo em sua velhice. Sendo assim, é importante analisar estudos que discutam as condições favoráveis para manter a capacidade para o trabalho dos indivíduos, a fim de promover ações de manutenção ou de prevenção de sua perda.

Um estudo de coorte, realizado com uma 
amostra representativa da população trabalhadora da Dinamarca, mostrou a importância de se avaliar a capacidade para o trabalho no sentido de prever os casos de absenteísmo por doença em longo prazo e aposentadoria precoce. Esse achado mostrou que a capacidade para o trabalho é um parâmetro viável para medir a capacidade das pessoas de continuar a fazer parte da força ativa de trabalho (SELL, 2009). Moura et al. (2013) destacam, ainda, que a promoção da capacidade funcional dos trabalhadores, ao longo dos anos das atividades laborais, pode contribuir para a qualidade de vida na aposentadoria, com redução dos custos para manutenção da saúde dos idosos.

No entanto, no Brasil, há um complicador importante relacionado ao fato de que não existia no País um sistema nacional que consolidasse informações sobre a saúde do servidor público, o que dificultava avaliar o perfil epidemiológico dessa categoria e inibia a elaboração de projetos de promoção da saúde de maior abrangência. Além disso, as estatísticas oficiais dos acidentes e das doenças associadas ao trabalho, fornecidas pela previdência social, eliminam da contagem todos os funcionários públicos estatutários civis e militares vinculados à União, aos estados e aos municípios, dificultando, portanto, as ações de prevenção de agravos e promoção da saúde para esse grupo de trabalhadores (CARNEIRO, 2006).

Segundo Carneiro (2006), os estudos na área pública brasileira têm possibilitado conhecer as condições de vida e de trabalho a que estão expostos os servidores públicos, a fim de analisar mais profundamente a relação saúde e trabalho. Integram parte do grupo de servidores públicos os quadros de servidores das universidades públicas compostos pelos docentes e os TécnicoAdministrativos em Educação (TAEs). Ressalta-se que a categoria dos TAEs pode ser de grande relevância científica na área da Saúde do Trabalhador, uma vez que engloba uma grande variedade de cargos dentro das diversas áreas de formação/atuação, abarcando cargos de níveis de escolaridade que vão desde a educação básica até o nível superior, mas, ainda assim, essa é uma população ainda pouco estudada.

Por essa razão, em 2012, foi iniciado um estudo de coorte com servidores TAEs de uma universidade pública federal, a fim de analisar sua capacidade para o trabalho e os fatores associados. Tal estudo mostrou que a maioria dos trabalhadores possuía condições de trabalho, de saúde e hábitos de vida favoráveis associados a uma alta taxa de boa ou ótima capacidade para o trabalho. No entanto, para mostrar se de fato esse quadro representava a realidade dessa população, uma das maneiras consideradas foi verificar, ao longo do tempo, quais indivíduos se aposentaram, o tipo de aposentadoria (por invalidez ou por tempo de serviço) e quais as características daqueles aposentados (GODINHO ET AL., 2016).

Com vistas a atender ao propósito desta investigação, buscou-se, portanto, analisar o perfil sociodemográfico, de trabalho e de saúde dos TAEs aposentados da Universidade Federal de Juiz de Fora (UFJF) diante do contexto atual de Reforma Previdenciária.

\section{Métodos}

O presente estudo foi desenvolvido por meio de um estudo de coorte realizado com a população de TAEs da UFJF. A população alvo foi composta por TAEs aposentados e a população de estudo pelos trabalhadores que se aposentaram no período de seguimento de quatro anos, compreendido entre os anos 2012/2013 a 2016/2017. Os dados foram coletados a partir dos registros do banco de dados daqueles servidores que participaram do estudo-base, em 2012/2013, e que, de acordo com os registros do vínculo com a universidade, aposentaram-se no período citado. 
Para caracterizar o perfil sociodemográfico dos servidores, foram analisadas as variáveis sexo, estado civil, cor, escolaridade e número de filhos; o perfil de trabalho foi apresentado pelas variáveis: idade com que começou a trabalhar, número de empregos, carga horária semanal, idade da aposentadoria, tempo de serviço total, tempo de serviço na UFJF, trabalho noturno, estresse no trabalho (demanda-controle) e apoio social no trabalho; já o perfil de saúde foi caracterizado pelas variáveis: ocorrência de sinais e sintomas de depressão, nível de atividade física, capacidade para o trabalho e, dentro deste último, o prognóstico próprio da capacidade para o trabalho. Para avaliar estas cinco últimas variáveis, foram utilizados instrumentos traduzidos, adaptados, testados e validados para o Brasil (ALVES ET AL., 2004; KROENKE; SPITZER; WILLIAMS, 2001; MATSUDO ET AL., 2001; SANTOS ET AL., 2013).

Para avaliar o estresse e o apoio social no trabalho, foi utilizada a escala Sueca Demanda-Controle-Apoio Social no Trabalho (DCS) (ALVES ET AL., 2004). A avaliação da ocorrência de sinais e sintomas de depressão foi realizada com a ajuda do Questionário sobre a Saúde do Paciente (PHQ-9), que consiste em um instrumento geral para rastreamento, diagnóstico, monitoramento e mensuração da severidade da depressão (KROENKE; SPITZER; WILLIAMS, 2001). O nível de atividade física foi avaliado através do Questionário Internacional de Atividade Física (Ipaq curto), que mede a atividade física relacionada à saúde na população. $\mathrm{O}$ Ipaq envolve quatro domínios de atividade física: relacionada ao trabalho, transporte, trabalho em casa e tempo de atividades de lazer. Esse questionário também inclui questões sobre tempo gasto sentado como um indicador de comportamento sedentário (SEGHETTO; PICCOLI, 2012). Por fim, foi avaliada a capacidade para o trabalho por meio do Índice de Capacidade para o Trabalho (ICT), instrumento desenvolvido por pesquisadores finlandeses, no qual a mensuração é baseada na autopercepção do trabalhador. O ICT foi previamente traduzido e adaptado para o Brasil por pesquisadores de universidades do estado de São Paulo e, posteriormente, foi validado por um estudo realizado com trabalhadores de uma companhia elétrica também no estado de São Paulo, Brasil (MARTINEZ ET AL., 2009; TUOMI ET AL., 2005).

A análise dos dados foi realizada por meio do software Statistical Package for the Social Sciences ${ }^{\circledR}$, através de estatística descritiva e inferencial. O estudo foi aprovado pelo Comitê de Ética em Pesquisa da Escola Nacional de Saúde Pública (CEP/ Ensp) e pelo Comitê de Ética em Pesquisa da Universidade Federal de Juiz de Fora, sob os números de parecer 1.574.457 e 1.673.735, respectivamente.

\section{Resultados}

Dos 833 servidores que compuseram a coorte, acompanhada desde 2012/2013, 88 aposentaram-se até fevereiro de 2017, sendo que, destes, apenas quatro $(4,5 \%)$ aposentaram-se por invalidez, comparados aos 95,5\% restantes que se aposentaram voluntariamente. Vale ressaltar que, em 2012/2013, do total de servidores participantes do estudo, $16,6 \%$ apresentavam capacidade para o trabalho baixa ou moderada. Os dados apresentados na tabela 1 denotam que estes aposentados são compostos, em sua maioria, por indivíduos do sexo masculino (52,9\%), casados $(62,1 \%)$, da cor branca $(65,5 \%)$, com nível de escolaridade universitário ou mais (61,2\%), média de 2,08 filhos, variando de 01 a $05(\mathrm{DP}=0,87)$, sendo que a maioria dos servidores que tinham filhos possuía até dois filhos (54,7\%). Vale ressaltar que $27,9 \%$ dos servidores não tinham filhos. 
Tabela 1. Características sociodemográficas, do trabalho e de saúde dos trabalhadores técnico-administrativos em educação da UFJF

\begin{tabular}{|c|c|c|c|c|c|c|c|}
\hline \multirow{3}{*}{ Variáveis } & \multirow{2}{*}{\multicolumn{2}{|c|}{ Total }} & \multicolumn{5}{|c|}{ Tipo de aposentadoria } \\
\hline & & & \multicolumn{2}{|c|}{ Por tempo de serviço } & \multicolumn{2}{|c|}{ Por invalidez } & \multirow[t]{2}{*}{$p$ valor } \\
\hline & $\mathrm{n}$ & $\%$ & $\mathrm{n}$ & $\%$ & $\mathrm{n}$ & $\%$ & \\
\hline Sexo & & & & & & & 0,364 \\
\hline Feminino & 41 & 47,1 & 40 & 97,6 & 01 & 2,4 & \\
\hline Masculino & 46 & 52,9 & 43 & 93,5 & 03 & 6,5 & \\
\hline Estado civil & & & & & & & 0,585 \\
\hline Casados & 54 & 62,1 & 51 & 94,4 & 03 & 5,6 & \\
\hline Não casados & 33 & 37,9 & 32 & 97,0 & 01 & 3,0 & \\
\hline Cor/raça & & & & & & & 0,504 \\
\hline Brancos & 57 & 65,5 & 55 & 96,5 & 02 & 3,5 & \\
\hline Não brancos & 30 & 34,5 & 28 & 93,3 & 02 & 6,7 & \\
\hline Escolaridade & & & & & & & 0,638 \\
\hline Universitário ou mais & 52 & 61,2 & 50 & 96,2 & 02 & 3,8 & \\
\hline Até ensino médio & 33 & 38,8 & 31 & 93,9 & 02 & 6,1 & \\
\hline Filhos & & & & & & & 0,588 \\
\hline Nenhum & 24 & 27,9 & 23 & 95,8 & 01 & 4,2 & \\
\hline Até 2 & 47 & 54,7 & 44 & 93,6 & 03 & 6,4 & \\
\hline 3 ou mais & 15 & 17,4 & 15 & 100 & 00 & 0,0 & \\
\hline Idade início do trabalho & & & & & & & 0,866 \\
\hline$>18$ anos & 46 & 54,1 & 44 & 95,7 & 02 & 4,3 & \\
\hline$<18$ anos & 39 & 45,9 & 37 & 94,9 & 02 & 5,1 & \\
\hline Número de empregos & & & & & & & 0,004 \\
\hline Um & 80 & 90,9 & 78 & 97,5 & 02 & 2,5 & \\
\hline Dois ou mais & 08 & 9,1 & 06 & 75,0 & 02 & 25,0 & \\
\hline Carga horária semanal & & & & & & & 0,676 \\
\hline$\leq 40$ horas & 67 & 82,7 & 64 & 95,5 & 03 & 4,5 & \\
\hline$>40$ horas & 14 & 17,3 & 13 & 92,9 & 01 & 7,1 & \\
\hline Idade da aposentadoria & & & & & & & 0,233 \\
\hline Até 60 anos & 62 & 74,7 & 58 & 93,5 & 04 & 6,5 & \\
\hline Mais de 60 anos & 21 & 25,3 & 21 & 100 & 00 & 0,0 & \\
\hline Tempo de trabalho total & & & & & & & 0,910 \\
\hline$<30$ anos & 02 & 2,5 & 02 & 100 & 00 & 0,0 & \\
\hline 31 a 40 anos & 38 & 46,9 & 36 & 94,7 & 02 & 5,3 & \\
\hline 41 a 50 anos & 34 & 42 & 32 & 94,1 & 02 & 5,9 & \\
\hline$>50$ anos & 07 & 8,6 & 07 & 100 & 00 & 0,0 & \\
\hline Tempo de trabalho na UFJF & & & & & & & 0,003 \\
\hline$<15$ anos & 02 & 2,3 & 01 & 50,0 & 01 & 50,0 & \\
\hline 21 a 30 a & 25 & 28,4 & 23 & 92,0 & 02 & 8,0 & \\
\hline$>30$ anos & 61 & 69,3 & 60 & 98,4 & 01 & 1,6 & \\
\hline Trabalho à noite & & & & & & & 0,613 \\
\hline Sim & 05 & 5,7 & 05 & 100 & 00 & 0,0 & \\
\hline Não & 82 & 94,3 & 78 & 95,1 & 04 & 4,9 & \\
\hline
\end{tabular}


Tabela 1. (cont.)

\begin{tabular}{|c|c|c|c|c|c|c|c|}
\hline Modelo demanda-controle & & & & & & & 0,809 \\
\hline Baixa exigência & 40 & 46,5 & 38 & 95,0 & 02 & 5,0 & \\
\hline Trabalho ativo & 07 & 8,1 & 07 & 100 & 00 & 0,0 & \\
\hline Trabalho passivo & 31 & 36,0 & 29 & 93,5 & 02 & 6,5 & \\
\hline Alta exigência & 08 & 9,3 & 08 & 100 & 00 & 0,0 & \\
\hline Apoio Social no Trabalho & & & & & & & 0,818 \\
\hline Baixo & 18 & 20,5 & 17 & 94,4 & 01 & 5,6 & \\
\hline Alto & 70 & 79,5 & 67 & 95,7 & 03 & 4,3 & \\
\hline Sinais e sintomas de depressão & & & & & & & 0,580 \\
\hline Ausente & 82 & 93,2 & 95,1 & 92,9 & 04 & 4,9 & \\
\hline Presente & 06 & 6,8 & 100 & 7,1 & 00 & 0,0 & \\
\hline Atividade Física & & & & & & & 0,878 \\
\hline Ativo/muito ativo & 50 & 56,8 & 48 & 96,0 & 02 & 4,0 & \\
\hline Pouco ativo & 24 & 27,3 & 23 & 95,8 & 01 & 4,2 & \\
\hline Sedentário & 14 & 15,9 & 13 & 92,9 & 01 & 7,1 & \\
\hline Capacidade para o Trabalho & & & & & & & 0,657 \\
\hline Ótima & 20 & 23 & 20 & 100 & 00 & 0,0 & \\
\hline Boa & 46 & 52,9 & 43 & 93,5 & 03 & 6,5 & \\
\hline Moderada & 17 & 19,5 & 16 & 94,1 & 01 & 5,9 & \\
\hline Baixa & 04 & 4,6 & 04 & 100 & 00 & 0,0 & \\
\hline \multicolumn{7}{|c|}{ Prognóstico próprio da capacidade para o trabalho } & 0,559 \\
\hline É improvável & 03 & 3,4 & 03 & 100 & 00 & 0,0 & \\
\hline Não estou certo & 24 & 27,3 & 22 & 91,7 & 02 & 8,3 & \\
\hline Bastante provável & 61 & 69,3 & 59 & 96,7 & 02 & 3,3 & \\
\hline
\end{tabular}

Fonte: Elaboração própria.

Quanto aos aspectos relacionados ao trabalho, foi encontrado que a maioria dos indivíduos aposentados começou a trabalhar depois de completar 18 anos (54,1\%) e que se aposentou com idade inferior a 60 anos (74,7\%). Desde que começaram a trabalhar, e até se aposentarem, 46,9\% trabalharam de 31 a 40 anos, sendo que, na UFJF, a maioria trabalhou 30 anos ou mais (69,3\%). Antes de se aposentar, a maioria dos servidores possuía apenas um emprego (90,9\%), trabalhava até 40 horas semanais (82,7\%), não trabalhava em trabalho de turno noturno (94,3\%), apresentava trabalho de baixa exigência $(46,5 \%)$ e alto apoio social no trabalho $(79,5 \%)$.
Pode-se observar na tabela 2 que a média de idade com que os servidores começaram a trabalhar foi de 17 anos, variando de 06 a 27 anos (DP= 4,65); a idade média de aposentadoria foi de 58,2 anos, variando de 50 a 74 anos (DP=4,48); a média de tempo de serviço, desde que começaram a trabalhar até a aposentadoria, foi de 41,2 anos, variando entre 27 e 61 anos (DP=6,32), sendo que, especificamente na UFJF, o tempo de serviço médio foi de 31 anos, variando de 08 a 40 anos (DP= $4,94)$; a carga horária semanal variou entre 20 e 70 horas semanais, com uma média de 37,2 horas $(\mathrm{DP}=8,46)$. 
Tabela 2. Médias das características do trabalho dos trabalhadores técnico-administrativos em educação da UFJF

\begin{tabular}{lrrrr}
\hline \multirow{2}{*}{ Características do trabalho dos trabalhadores } & \multicolumn{2}{c}{ Médias } & DP \\
\cline { 2 - 4 } Idade com que começou a trabalhar & Média & Mínimo & Máximo & \\
Idade com que aposentou & 17,0 & 06 & 27 & 4,65 \\
Tempo de trabalho total & 58,2 & 50 & 74 & 4,48 \\
Tempo de trabalho na UFJF & 41,2 & 27 & 61 & 6,32 \\
Carga horária semanal & 31,0 & 08 & 40 & 4,94 \\
Número de filhos & 37,2 & 20 & 70 & 8,46 \\
\hline
\end{tabular}

Fonte: Elaboração própria.

Com relação aos aspectos relacionados à saúde, a maioria dos indivíduos não apresentava sinais e sintomas de depressão (93,2\%); $56,8 \%$ foram classificados como ativos ou muito ativos no que diz respeito ao nível de atividade física; e 52,9\% apresentaram boa capacidade para o trabalho. Quanto ao prognóstico próprio da capacidade para o trabalho, foi realizada a seguinte pergunta: 'Considerando sua saúde, você acha que será capaz, daqui a 2 anos, de fazer seu trabalho atual?'. Foram oferecidas três opções de respostas: 'É improvável', 'Não estou muito certo' ou 'Bastante provável'. Dos 88 indivíduos aposentados, a maioria respondeu que seria 'Bastante provável' (69,3\%).

Ao analisar, especificamente, os quatro servidores que se aposentaram por invalidez, pode-se perceber que eles eram, em sua maioria, homens (75\%), casados (75\%), possuíam até 02 filhos (75\%), trabalhavam 40 horas semanais ou menos (75\%), aposentaram-se antes de completar 60 anos de idade (100\%), trabalharam de 20 a 30 anos na UFJF (50\%), não trabalhavam à noite (100\%), possuíam alto apoio social no trabalho (75\%), não apresentavam sinais e sintomas de depressão (100\%), eram ativos ou muito ativos quanto à realização de atividade física (50\%) e apresentavam boa capacidade para o trabalho (75\%). As demais variáveis de estudo não apresentaram diferenças significativas, sendo que, como exemplo, pode-se citar o resultado encontrado para a avaliação do prognóstico próprio da capacidade para o trabalho. Neste caso, metade dos aposentados por invalidez responderam que em dois anos seria 'Bastante provável' que seriam capazes de continuar trabalhando, ao mesmo tempo que a outra metade dos servidores não estava muito certa se seria capaz de continuar realizando seu trabalho; e ninguém respondeu que seria improvável continuar trabalhando.

Com relação às doenças diagnosticadas na população de estudo, conforme pode ser observado no gráfico 1, tem-se que as mais prevalentes foram as doenças musculoesqueléticas, como artrose, bico de papagaio, problemas na coluna, hérnia de disco, Lesão por Esforço Repetitivo (LER) e dor lombar; doenças neurológicas ou dos órgãos dos sentidos, como depressão (leve e severa), ansiedade, enxaqueca e glaucoma; hipertensão arterial; doenças gastrintestinais, como gastrite, refluxo e hérnia de hiato; e doenças respiratórias, como rinite alérgica e Doença Pulmonar Obstrutiva Crônica (DPOC). 
Gráfico 1. Quantitativo de doenças diagnosticadas entre os técnico-administrativos em educação da UFJF identificadas no período do estudo (2012/2013 a 2016/2017)

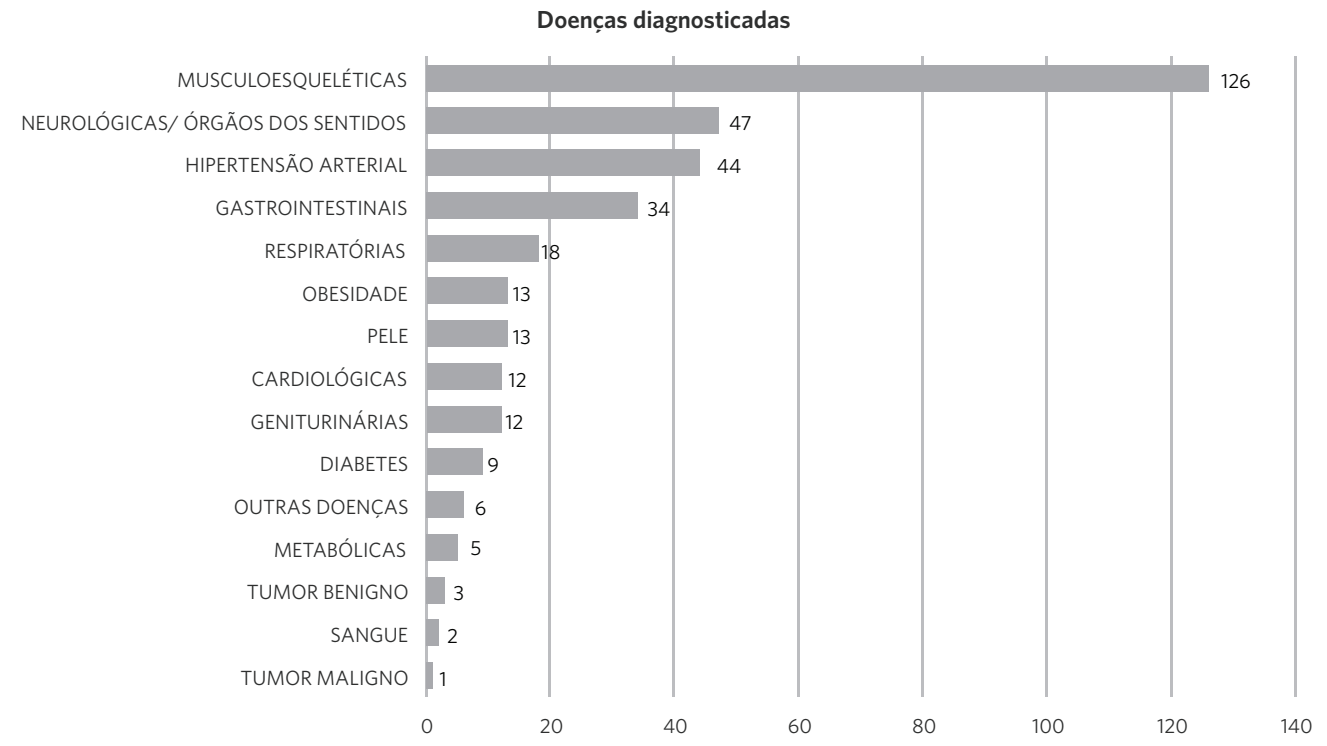

Fonte: Elaboração própria.

Ao se realizar a análise bivariada, utilizando como variável desfecho o tipo de aposentadoria (voluntária $\mathrm{x}$ invalidez), pôde-se observar não houve diferenças estatisticamente significativas entre os grupos estudados.

\section{Discussão}

Nessa investigação, os resultados mostraram que a população estudada apresentou características sociodemográficas e ocupacionais diferentes da população geral, principalmente com relação ao tipo de vínculo com a instituição. Isso porque os TAEs têm estabilidade no emprego, o que se configura como uma situação diferente da crescente instabilidade e terceirização que vem acontecendo em muitos setores no Brasil nos últimos anos, situações que impactam profundamente as condições de trabalho da população, as relações de trabalho, além de gerarem a precarização que configura o mundo do trabalho atualmente e que vem se acentuando com as reformas que estão ocorrendo no que diz respeito às leis trabalhistas e aos direitos conquistados pelos trabalhadores ao longo dos anos (LAZZARESCHI, 2015).

Não se pode deixar de levar em consideração que o perfil favorável apresentado pelos TAEs com relação a diversos aspectos pode ser consequência do efeito do trabalhador saudável, sendo importante notar que o bom resultado do perfil de saúde identificado nos trabalhadores participantes pode estar relacionado a essa questão. O efeito do trabalhador saudável é discutido por diversos autores que já realizaram estudos sobre o perfil de saúde no local de trabalho, e esses autores sempre discutem a importância de analisar cuidadosamente tal efeito ao discutir os resultados dos estudos (GRIEP ET AL., 2005; KROENKE; SPITZER; WILLIAMS, 2001; MATSUDO ET AL., 2001).

Para discutir o perfil dos indivíduos que se aposentaram na universidade é importante ressaltar a relação que Sell (2009) faz entre aposentadoria-absenteísmo-aposentadoria precoce. A autora analisou em seu estudo 
a relação entre capacidade para o trabalho autorrelatada, absenteísmo por doença em longo prazo e aposentadoria precoce, e encontrou associação significativa entre as variáveis. Os resultados de seu estudo mostraram que a redução de um ponto na percepção da capacidade para o trabalho, em uma escala de dez pontos, foi associada ao aumento de $15,1 \%$ do risco de absenteísmo por doença em longo prazo e aumento de $33 \%$ do risco de aposentadoria precoce. A autora relata que a razão de chance maior para aposentadoria precoce, comparada com o absenteísmo por doença, era esperada, uma vez que a reduzida capacidade para o trabalho é considerada a principal razão de os indivíduos se aposentarem antes do tempo. Portanto, levar-se-á em consideração esse achado para embasar a discussão dos resultados encontrados no presente estudo ao realizar a análise do perfil dos aposentados, tanto dos que se aposentaram voluntariamente quanto daqueles que se aposentaram por invalidez.

Entre os TAEs aposentados, pode-se observar que, embora tenha havido uma porcentagem de indivíduos com baixa ou moderada capacidade para o trabalho, esse não foi um fator determinante para o indivíduo se aposentar precocemente. As características sociodemográficas mostraram que, mesmo tendo havido uma distribuição proporcional de homens e mulheres (52,9\% e $47,1 \%$, respectivamente), houve uma prevalência maior de homens entre os indivíduos aposentados. Embora no presente estudo não tenha sido encontrada diferença estatisticamente significativa, não se pode deixar de levar em consideração o que se discute na literatura com relação ao fato de que há uma chance maior de as mulheres terem capacidade mais baixa para o trabalho em relação aos homens. Isso porque as demandas do ambiente doméstico podem ocasionar jornadas de trabalho duplas e até mesmo triplas, que fazem as trabalhadoras exercerem papéis adicionais aos de sua profissão, como o de mãe e esposa. De acordo com os autores, essa situação pode levar a um gasto de energia e de tempo que poderia ser convertido em atividades físicas, de lazer ou sociais, que promovem a saúde e a capacidade para o trabalho (HILLESHEIN ET AL., 2011).

Houve predominância, também, de indivíduos da cor branca e casados ou em união estável. No entanto, essas variáveis também não se associaram estatisticamente à perda da capacidade para o trabalho ou à situação de aposentadoria. No que diz respeito à escolaridade, a maioria dos aposentados possuía nível superior ou mais. Moura et al. (2013) encontraram resultados semelhantes aos do presente estudo ao realizarem uma pesquisa sobre capacidade para o trabalho de funcionários da prefeitura de um campus universitário público. Eles obtiveram a prevalência de trabalhadores com nível de escolaridade universitário ou mais (57,7\%). Esses autores discutem que níveis mais altos de escolaridade são facilitadores para ações de educação em saúde, na medida em que estimulam o indivíduo na busca de hábitos de vida mais saudáveis, que contribuem para a melhora da capacidade para o trabalho e, consequentemente, reduzem as chances de aposentadoria precoce, mas essa variável também não foi associada ao tipo de aposentadoria na presente investigação.

Quanto aos aspectos relacionados ao trabalho, as variáveis analisadas não obtiveram associação significativa com relação à aposentadoria dos indivíduos estudados. No entanto, há de se considerar aspectos importantes já discutidos na literatura relacionados a algumas variáveis analisadas aqui. Quanto ao número de empregos, por exemplo, a maioria dos indivíduos analisados no presente estudo possuía apenas um emprego. Benavides et al. (2015) discutem em seu estudo que o alto número de contratos de trabalho associou-se à aposentadoria precoce devido à incapacidade permanente, mostrando que é mais recomendável que os trabalhadores mantenham apenas um vínculo empregatício para prevenir danos 
futuros à sua saúde e à capacidade para o trabalho. Quanto à carga horária semanal, a Constituição brasileira de 1988 estipula o direito dos trabalhadores a trabalhar por um período não superior a oito horas diárias e quarenta e quatro horas semanais. Assim, pode-se verificar que os técnico-administrativos aposentados da UFJF seguiam essa carga horária adequadamente. No entanto, também não houve associação significativa entre essa variável e o tipo de aposentadoria.

Quanto ao apoio social no trabalho, este se refere à interação entre colegas e chefias na cooperação para a realização do trabalho. Negeliskii e Lautert (2011) consideram que o apoio social deve ser a base das relações de trabalho e uma estratégia de organização social nas instituições, pois dessa forma se obtém a redução e, até mesmo, a prevenção do estresse laboral. Ao se valorizar as relações e o ambiente de trabalho, são promovidos benefícios à saúde dos trabalhadores e à capacidade para o trabalho. No presente estudo, tanto aqueles que se aposentaram voluntariamente quanto os que se aposentaram por invalidez possuíam alto apoio social no trabalho.

Com relação à saúde, pode-se notar que os aposentados da universidade apresentaram um perfil favorável. No entanto, alguns servidores ainda relataram ter algum tipo de dor, doença ou lesão, sendo as doenças prevalentes semelhantes às encontradas por outros autores. Eles discutem que as doenças mentais e musculoesqueléticas representaram a maior carga de doença nos indivíduos em situação de incapacidade permanente em seu estudo (López et AL., 2014). Porém, mesmo apresentando alguns tipos de doenças, dores ou lesões, a maioria dos trabalhadores que se aposentaram não apresentava sinais e sintomas de depressão, teve boa classificação quanto ao nível de atividade física e apresentou boa capacidade para o trabalho. Embora nenhuma dessas variáveis tenha apresentado associação significativa com o tipo de aposentadoria dos indivíduos no presente estudo, não se deve deixar de dar atenção aos indivíduos com sintomas depressivos, sendo esse um fator importante de ser avaliado nos trabalhadores, no sentido de prevenir que se aposentem precocemente devido a transtornos mentais como a depressão.

Como parte das medidas propostas para prevenir o adoecimento do trabalhador ao longo de sua vida laboral, os hábitos de vida saudáveis, como a prática regular de atividades físicas, devem ser estimulados. Robroek et al. (2013) trazem uma contribuição importante em seu estudo quando mostram que a falta de atividade física durante o tempo de lazer pode aumentar o risco de aposentadoria por incapacidade. De fato, pode-se observar que, entre os técnico-administrativos do presente estudo, dois dos que se aposentaram por invalidez foram classificados como pouco ativos ou sedentários. Portanto, conforme discutido anteriormente em outro estudo (GODINHO ET AL., 2016), os hábitos de vida saudáveis devem ser estimulados juntamente com a reorganização do ambiente de trabalho, no sentido de promover a capacidade para o trabalho e prevenir as aposentadorias precoces.

É importante ressaltar que, no presente estudo, nenhuma variável estudada apresentou associação significativa com o tipo de aposentadoria (aposentadoria voluntária x aposentadoria por invalidez). Quanto a essa questão, podem ser levantadas duas hipóteses: a primeira está relacionada ao tamanho da amostra, refletido na pequena porcentagem de indivíduos aposentados por invalidez; a segunda se deve ao fato de que os trabalhadores, de uma maneira geral, apresentaram condições de trabalho e saúde favoráveis, não sendo, portanto, tais variáveis determinantes para o tipo de aposentadoria desses trabalhadores. Sendo assim, o presente estudo possui duas limitações principais: o tamanho pequeno da amostra e também o fato de não ter sido possível ter acesso à informação sobre o motivo da aposentadoria por invalidez dos quatro trabalhadores relatados acima. Dessa forma, não se pode afirmar que o motivo da incapacidade desses 
trabalhadores tenha sido relacionado ao trabalho ou à saúde especificamente. Ainda assim, mesmo diante das limitações desta pesquisa, baseando-se nas boas condições de trabalho e saúde que esses indivíduos apresentavam, supõe-se que a incapacidade que os levou a se aposentar precocemente, possivelmente, não tenha sido associada ao trabalho.

\section{Conclusões}

Pode-se concluir que, de fato, os trabalhadores da UFJF possuem, predominantemente, um perfil de trabalho e de saúde que pareceu ser satisfatório para a manutenção da vida ativa no trabalho, situação retratada no alto percentual de aposentadorias por idade ou tempo de serviço e no baixo percentual de aposentadorias por invalidez. Embora nenhuma variável tenha se associado ao tipo de aposentadoria, e esses resultados devam ser extrapolados para a população geral brasileira com cautela, pois se referem a uma população muito específica, os resultados encontrados podem servir, juntamente a evidências já apresentadas em outros estudos, como base para as discussões acerca das condições de trabalho e saúde que favorecem que o trabalhador não tenha prejuízo na sua capacidade para o trabalho e, consequentemente, não se aposente precocemente.

Sugere-se, portanto, que, antes de se concretizarem as ações propostas na Reforma da Previdência, sejam discutidas mais amplamente medidas que visem a proporcionar aos trabalhadores boas condições de trabalho e de saúde, como remuneração justa, a fim de evitar que o trabalhador precise de mais de um vínculo empregatício para complementar sua renda familiar; favorecer a boa interação dos trabalhadores com os colegas e as chefias visando a aumentar o apoio social no trabalho; além de proporcionar momentos em que os trabalhadores possam desenvolver hábitos de vida saudáveis, como a prática de atividades físicas, que promovem a saúde e, ao mesmo tempo, previnem o adoecimento, a perda da capacidade para o trabalho e as aposentadorias precoces.

Assim, as discussões que envolvem a Reforma Previdenciária vão muito além da proposta e da implementação de mudanças que farão com que os trabalhadores permaneçam por mais tempo no mercado de trabalho. Muito mais que isso, sugere-se que sejam propostas ações integradas de atenção à saúde do trabalhador ao longo da vida laboral focadas na prevenção de agravos e na promoção da saúde, de modo que o trabalhador permaneça no mercado de trabalho, sim, mas com saúde, dignidade e qualidade de vida.

\section{Colaboradores}

Os autores participaram da concepção, do planejamento, da análise, da interpretação dos dados e da aprovação da versão final do manuscrito.

\section{Agradecimentos}

Nossos mais sinceros agradecimentos aos servidores técnico-administrativos em educação, sem os quais nosso estudo não teria se concretizado. 


\section{Referências}

AGUIAR, L.; VASCONCELLOS, L. C. F. A gestão do Sistema Único de Saúde e a Saúde do Trabalhador: O direito que se tem e o direito que se perde. Saúde em Debate, Rio de Janeiro, v. 39, n. 106, p. 830-840, 2015.

AKIYAMA, H. Japan's longevity challenge. Science, [S. 1.], v. 350, n. 6265 , p. 1135-1135, 2015.

ALVES, M. G. M. et al. Versão resumida da job stress scale: adaptação para o português. Revista de Saúde Pública, São Paulo, v. 38, n. 2, p. 164-171, 2004.

BARILE, B. et al. Activities of daily living, chronic medical conditions, and health-related quality of life in older adults. The Journal of Ambulatory Care Management, Germantown, v. 35, n. 4, p. 292-303, 2012.

BENAVIDES, F. G. et al. Labour market trajectories and early retirement due to permanent disability: a study based on 14972 new cases in Spain. The European Journal of Public Health, Oxford, v. 25, n. 4, p. 673-677, ago. 2015.

CARNEIRO, S. A. M. Saúde do trabalhador público: questão para a gestão de pessoas - a experiência na Prefeitura de São Paulo. Revista do Serviço Público, Brasília, DF, v. 57, n. 1, p. 23-49, 2006.

FREIRE, F. S.; MENDONÇA, L. H.; COSTA, A. J. B. Sustentabilidade econômica das instituições de longa permanência para idosos. Saúde em Debate, Rio de Janeiro, v. 36, n. 95, p. 533-543, 2012.

GODINHO, M. R. et al. Work ability and associated factors of Brazilian technical-administrative workers in education. BMC Research Notes, Londres, v. 9, n. 1, dez. 2016.

GRIEP, R. H. et al. Validade de constructo de escala de apoio social do Medical Outcomes Study adaptada para o português no Estudo Pró-Saúde. Cadernos de Saúde Pública, Rio de Janeiro, v. 21, n. 3, p. 703-714, 2005.

HILLESHEIN, E. F. et al. Capacidade para o trabalho de enfermeiros de um hospital universitário. Revista
Gaúcha de Enfermagem, Porto Alegre, v. 32, n. 3, p. 509515, 2011.

KROENKE, K.; SPITZER, R. L.; WILLIAMS, J. B. The PHQ-9: validity of a brief depression severity measure. Journal of General Internal Medicine, Philadelphia, v. 16, n. 9, p. 606-613, 2001.

LAZZARESCHI, N. A aposentadoria no contexto das transformações das condições de trabalho. Revista Kairós Gerontologia, São Paulo, v. 18, n. 3, p. 29-56, 2015.

LIMA-COSTA, M. F.; VERAS, R. Saúde pública e envelhecimento. Cadernos de Saúde Pública, Rio de Janeiro, v. 19, p. 700-701, 2003.

LÓPEZ, M. A. et al. Estimación de la carga de enfermedad por incapacidad laboral permanente en España durante el período 2009-2012. Revista Española de Salud Pública, Madrid, v. 88, n. 3, p. 349-358, jun. 2014.

MARTINEZ, M. C. et al. Validade e confiabilidade da versão brasileira do Índice de Capacidade para o Trabalho. Revista de Saúde Pública, São Paulo, v. 43, n. 3, p. 525-532, 2009.

MATSUDO, S. et al. Questionário Internacional de Atividade Física (IPAQ): estudo de validade e reprodutibilidade no Brasil. Revista Brasileira de Atividade Física \&t Saúde, Pelotas, v. 6, n. 2, p. 5-18, 2001.

MOURA, A. L. et al. Capacidade para o trabalho de funcionários da prefeitura de um campus universitário público. Revista Eletrônica de Enfermagem, Goiânia, v. 15, n. 1, p. 130-137, 2013.

NEGELISKII, C.; LAUTERT, L. Estresse laboral e capacidade para o trabalho de enfermeiros de um grupo hospitalar. Revista Latino-Americana de Enfermagem, Ribeirão Preto, v. 19, n. 3, p. 1-8, 2011.

ROBROEK, S. J. et al. The contribution of overweight, obesity, and lack of physical activity to exit from paid employment: a meta-analysis. Scandinavian Journal of Work, Environment \& Health, Helsinki, v. 39, n. 3, p. 
233-240, maio 2013.

SANTOS, I. S. et al. Sensibilidade e especificidade do Patient Health Questionnaire-9 (PHQ-9) entre adultos da população geral. Cadernos de Saúde Pública, Rio de Janeiro, v. 29, n. 8, p. 1533-1543, 2013.

SANTOS, T. R. et al. Perfil socioeconômico-demográfico do beneficiário do Instituto Nacional do Seguro Social aposentado por invalidez e suas causas, no Estado da Paraíba, no quinquênio 2007-2011. Revista Brasileira de Estudos de População, Rio de Janeiro, v. 29, n. 2, p. 349-359, 2012.

SEGHETTO, A.; PICCOLI, J. C. J. Nível de atividade física, prevalência de desconforto e dor muscular e capacidade de trabalho: uma avaliação no setor de call center de um banco do Rio Grande do Sul, Brasil. Revista Brasileira de Ciência e Movimento, Taguatinga, v. 20, n. 3, p. 105-117, 2012.
SELL, L. Predicting long-term sickness absence and early retirement pension from self-reported work ability. International Archives of Occupational and Environmental Health, Berlin, v. 82, n. 9, p. 1133-1138, out. 2009.

TUOMI, K. et al. Índice de capacidade para o trabalho. São Carlos: EduFSCar, 2005.

YORKSTON, K. M.; BOURGEOIS, M. S.; BAYLOR, C. R. Communication and Aging. Physical Medicine and Rehabilitation Clinics of North America, Filadélfia, v. 21, n. 2, p. 309-319, 2010.

Recebido para publicação em agosto de 2017

Versão final em outubro de 2017

Conflito de interesses: inexistente

Suporte financeiro: apoio financeiro do Programa de Excelência Acadêmica da Coordenação de Aperfeiçoamento de Pessoal de Nível Superior (Proex-Capes) do Programa de Pós-Graduação em Saúde Pública da Escola Nacional de Saúde Pública (Ensp) da Fundação Oswaldo Cruz (Fiocruz) 